\title{
Potencial erosivo de jugos de frutas amazónicas
}

\author{
Erosive potential of juices of amazonian fruits
}

\author{
Shareen Melannie Acuña Flores ${ }^{1, a, b}$, Lidia Yileng Tay Chu Jon ${ }^{2, c, d}$, Renzo Alberto Ccahuana-Vasquez ${ }^{3, d, e}$, \\ Leyla Delgado-Cotrina ${ }^{2, b, c}$.
}

\begin{abstract}
RESUMEN
La erosión dental es la pérdida irreversible de la superficie del esmalte o dentina, debido a exposición frecuente de ácidos de origen no bacteriano. A pesar de que ciertos jugos de frutas presentan potencial erosivo in vitro, no existe una evidencia conclusiva al respecto. Objetivos: Evaluar in situ el potencial erosivo de jugos de frutas amazónicas sobre el esmalte dental. Material y métodos: Se evaluó in situ la microdureza superficial Vickers (MSV) del esmalte dental luego de la exposición a las bebidas Physalis Peruviana (aguaymanto), Averrhoa Carambola (carambola), Myrciaria Dubia (camu camu), Coca cola (control positivo) y agua destilada (control negativo). Participaron quince voluntarios (18 a 30 años) quienes utilizaron placas palatinas para reproducir el medio de almacenamiento. Se colocaron 6 fragmentos por placa. Las placas fueron expuestas a las diferentes bebidas $4 \mathrm{v} / \mathrm{d}(9: 00 \mathrm{am}, 11: 00 \mathrm{am}, 13: 00 \mathrm{pm}$ y 15:00 pm) durante 5 minutos por 5 días. Por cada ciclo erosivo se expuso a dos bebidas, inmediatamente los dispositivos palatinos fueron recolocados en la boca de los participantes. Hubo un periodo de washout de 8 días entre cada ciclo. Se calculó la diferencia de MSV antes y después de la exposición. El análisis de ANOVA/Tukey se utilizó para determinar las diferencias entre las bebidas en el programa SPSS 19, con un nivel de significancia de 0,5\%. Resultados: Las cuatro bebidas evaluadas disminuyeron la MSV del esmalte $(\mathrm{p}<0,05)$ sin diferencia significativa entre ellas. Conclusiones: Los jugos a base de camu camu, aguaymanto y carambola son potencialmente erosivos.
\end{abstract}

PALABRAS CLAVE: Erosión dental, jugos de frutas y vegetales, dureza.

\section{SUMMARY}

Dental erosion is the irreversible loss of the surface of the enamel or dentin, due to frequent exposure of nonbacterial acids. Although certain fruit juices have erosive potential in vitro, there is no conclusive evidence in this regard. Objective: To evaluate in situ the erosive potential of Amazonian fruit juices on dental enamel. Material and methods: The Vickers surface microhardness (VMH) of dental enamel was evaluated in situ after exposure to drinks Physalis Peruviana (aguaymanto), Averrhoa Carambola (carambola), Myrciaria Dubia (camu camu), Coca cola (positive control) and distilled water (negative control). Fifteen volunteers (18 to 30 years) participated who wore palatal appliances to reproduce the storage medium. Each one containing 6 enamel blocks. The appliances were exposed to the different drinks 4 times a day (9:00 am, 11:00 am, 13:00 pm and 15:00 pm) for 5 minutes during 5 days. For each erosive cycle, they were exposed to two drinks, immediately the palatal devices were repositioned in the participants' mouth. There was an 8-day washout period between each cycle. The difference in VMH before and after exposure was calculated. The ANOVA/Tukey analysis was used to determine the differences between beverages in the SPSS 19 program, with a significance level of $0.5 \%$. Results: The four experimental groups decreased enamel VMH $(\mathrm{p}<0.05)$ without significant difference between them. Conclusions: The juices based on camu camu, aguaymanto and carambola are potentially erosive. KEYWORDS: Tooth erosion, fruit and vegetable juices, hardness.

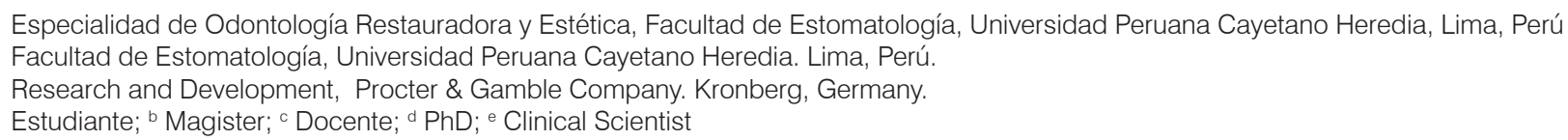




\section{INTRODUCCIÓN}

La erosión dental es una condición multifactorial que describe la pérdida química de la superficie de esmalte y dentina por exposición a ácidos no derivados de bacterias orales $(1,2)$. La prevalencia global de erosión dental en todo el mundo es de $30 \%$ a $50 \%$ en niños, $30 \%$ en adolescentes y de $20 \%$ a $45 \%$ en adultos $(3,4)$.

Los ácidos responsables de la erosión provienen de fuentes extrínsecas (dieta, hábitos de higiene, consumo de medicamentos, actividad física, ocupación) y fuentes intrínsecas (reflujo gastroesofágico, trastornos de la alimentación) (3-5). En los últimos años los hábitos dietéticos han cambiado, se ha incrementado el consumo de jugos y frutas naturales por su alto nivel de antioxidantes, fibras y vitaminas, sin embargo también han sido asociados a mayor prevalencia de erosión dental $(2,3,6)$. La ingesta de cítricos, el consumo de frutas más de dos veces al día, consumo diario de refrescos y consumo semanal de vinagre o bebidas deportivas puede aumentar significativamente el riesgo de erosión dental $(5,7)$. Asimismo, la mayor pérdida de minerales en los dientes se produce a un $\mathrm{pH}$ menor a 4,5 de tal forma que los jugos de frutas ( $\mathrm{pH} 2,78-4,60)$, bebidas carbonatadas $(\mathrm{pH} 2,5-3,46)$,

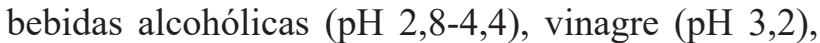
suplementos deportivos ( $\mathrm{pH} 3,17-3,87$ ) presentan un pH bajo (8-10).

Ciertas frutas y bebidas industrializadas contienen diferentes tipos de ácidos débiles que les confieren el potencial erosivo de las estructuras dentales, cuyas características dependen de factores químicos acidez titulable (AT), tipo de ácido, $\mathrm{pH}$, adhesión del producto a la superficie, presencia y concentración de calcio, fosfato, fluoruro; factores de comportamiento: forma de ingerir la bebida, consumo excesivo de alimentos o bebidas ácidas, exposición nocturna a bebidas ácidas, prácticas de higiene bucal y factores biológicos los que son aspectos inherentes al individuo: capacidad buffer, rango de flujo salival, formación de película adquirida, composición y estructura de los dientes, anatomía y oclusión, movimientos fisiológicos de los tejidos blandos $(5,8,10,11)$.

En la amazonía peruana existen frutas que son consumidas frecuentemente por sus propiedades antioxidantes y alto contenido de vitamina C. Veras en Brasil y Ruilova en Perú encontraron que el camu camu presenta un $\mathrm{pH}$ de 2,80 y 2,90 respectivamente, produciendo disminución de la microdureza superficial del esmalte in vitro $(12,13)$, sin embargo los resultados no se pueden replicar en el entorno oral con todas las variaciones biológicas que influyen en la erosión (14), mientras que un esquema de investigación in situ permite la posibilidad de controlar el desafío erosivo, permitiendo contacto directo con la saliva dando lugar a la formación de la película adquirida, siendo este último, un factor relevante en el desarrollo y progresión de las lesiones erosivas $(14,15)$. Por otro lado, no existe una evidencia conclusiva del potencial erosivo de los jugos de fruta (7).

El objetivo del presente estudio fue evaluar in situ el potencial erosivo de frutas amazónicas Physalis Peruviana (aguaymanto), Averrhoa Carambola (carambola), Myrciaria Dubia (camu camu) sobre el esmalte dental mediante microdureza superficial Vickers (MSV).

\section{MATERIAL Y MÉTODOS}

\section{Diseño experimental}

El presente estudio doble ciego, randomizado e in situ presentó 3 fases de 5 días con un intervalo de 8 días entre ellos. El protocolo fue aprobado por el Comité Institucional de Ética de la Universidad Peruana Cayetano Heredia ( $\mathrm{N}^{\circ}$ 477-20-18). Se incluyeron quince voluntarios entre 18 - 30 años (3 hombres y 12 mujeres) con buen estado general, flujo salival estimulado mayor a $1 \mathrm{ml} / \mathrm{min}$ y no estimulado mayor a $0,1 \mathrm{ml} / \mathrm{min}$. Se excluyeron de este estudio voluntarios con lesiones de caries activa, enfermedad periodontal, aparatología ortodóncica, fumadores, uso de medicamentos en los últimos 2 meses (antidepresivos, narcóticos, diuréticos, antihistamínicos, antimicrobianos), enfermedades sistémicas autoinmunes, xerostomía, diabetes tipo 1, malnutrición, problemas gastroesofágicos y regurgitación o vómito (16-21). Antes de la participación los voluntarios firmaron un consentimiento informado.

Para la evaluación del flujo salival estimulado y no estimulado se recolectó saliva 2 horas después de la última comida. Para la saliva estimulada, los voluntarios masticaron un pedazo de parafina (Parafilm ${ }^{\circledR}$ M, Illinois, Estados Unidos) durante un minuto, luego escupieron por 5 minutos en tubos 
cónicos de polipropileno de 17 x $120 \mathrm{~mm}(17,18,22)$. El tamaño de muestra fue calculado en base a un estudio piloto con 3 voluntarios. Se estimó un tamaño de muestra de 15 voluntarios basados un nivel de confianza del 95\%, poder estadístico de $85 \%$, considerando una deserción del $20 \%$ lo que corresponde a lo reportado en la literatura $(14,16-$ $18,21)$.

\section{Preparación de los especímenes}

Se utilizaron 225 especímenes de esmalte $(4 \times 4 \times$ $2 \mathrm{~mm}$ ) obtenido de premolares humanos sanos, sin signos de erosión, sin manchas, ni deformaciones, sin restauraciones ni grietas (16). Los dientes fueron seccionados con un disco diamantado biactivo (KG Sorensen $^{\circledR}$, São Paulo, Brasil) bajo refrigeración constante, para obtener especímenes de 4 × 4 × 2 $\mathrm{mm}$. El pulido de la superficie de esmalte se realizó con lija de agua (Asalite, Lima, Perú) de grano $600,800,1000,1500$ y 2000 durante 2 min con irrigación constante con cada lija, para lograr una superficie uniforme. Finalmente, los especímenes fueron expuestos a ultrasonido durante 10 minutos para eliminar los residuos. Todos los especímenes se almacenaron en agua destilada desionizada bajo refrigeración a una temperatura de $4^{\circ} \mathrm{C}$ hasta que se iniciaron los ciclos erosivos (19). Todas estas muestras fueron esterilizadas en óxido de etileno (1618). Se prepararon 45 especímenes para cada bebida evaluada.

\section{Confección de los dispositivos intraorales}

Se fabricó una placa palatina intraoral removible de resina acrílica autopolimerizable, por participante. Los especímenes fueron distribuidos aleatoriamente para cada sujeto y para cada fase del estudio para lo cual se realizaron 6 cavidades $(4 \times 4 \times 2 \mathrm{~mm}), 3$ de cada lado. Los especímenes fueron fijados a las cavidades con cera pegajosa en bastón (Asfer, São Paulo, Brasil). Adicionalmente, se colocaron dos alambres ortodónticos adheridos al dispositivo sobre las cavidades de la placa, sin contacto con el esmalte, para garantizar la ausencia de abrasión por la lengua (22). Todos los especímenes fueron colocados a nivel de la placa palatina y fueron reemplazados en cada fase experimental.

\section{Preparación de las bebidas}

Se emplearon las siguientes bebidas: Physalis Peruviana (aguaymanto), Averrhoa Carambola (carambola), Myrciaria Dubia (camu camu), Coca cola $^{\circledR}$ (control positivo) y agua destilada (control negativo). Para la preparación de los jugos de frutas se seleccionaron frutas maduras y en buen estado. Se lavaron en agua corriente y luego se secaron a temperatura ambiente. Para la preparación de los jugos se utilizaron $100 \mathrm{ml}$ de pulpa de fruta con 200 $\mathrm{ml}$ de agua destilada desionizada, en una proporción 1:2 dando un total de $300 \mathrm{ml}$ de jugo (23). Las bebidas se mantuvieron a temperatura ambiente y se utilizaron el mismo día (13).

\section{Ciclo erosivo}

Se entregó una placa palatina a los voluntarios junto a una lista de indicaciones diarias, agua destilada desionizada, cepillo dental Colgate $360^{\circ}$ (Colgate Palmolive S.A), pasta dental Colgate Máxima Protección (Colgate Palmolive S.A), recipiente plástico para almacenar las placas acrílicas y gasa. Los voluntarios realizaron su higiene dental normalmente, sin cepillar el dispositivo. También se informó a los voluntarios de no utilizar ningún producto fluorado o alimentos ricos en flúor durante el desarrollo del estudio.

Todos los voluntarios elegibles fueron identificados por un número único de estudio. Se les asignó al azar un orden de tratamiento de secuencia de acuerdo con el orden de entrada en el estudio. La asignación al azar se determinó a partir de una tabla de asignación estándar al azar. Los especímenes fueron distribuidos aleatoriamente a los diferentes grupos experimentales y codificados. La placa palatina fue instalada la noche anterior al inicio de la fase experimental, después de la última higiene y antes de dormir para propiciar la formación de película adquirida. El segundo día las placas acrílicas fueron expuestas a las bebidas, 4 veces al día en horarios predeterminados (9:00 am, 11: 00 am, 13:00 pm y 15:00 pm) de la siguiente forma: El dispositivo se retiró del paladar y fue colocado dentro de un recipiente que contenía una bebida durante 5 minutos, se procedió a lavar con agua destilada desionizada y se sumergió el otro lado del dispositivo en otra bebida durante 5 minutos, luego se procedió a lavar con agua destilada e inmediatamente fueron recolocados en la boca. Estos dispositivos fueron usados diariamente, inclusive para dormir, excepto durante los horarios de alimentación (desayuno, almuerzo y cena). Después de cada fase experimental hubo un periodo de washout de 8 días para evitar 
cualquier efecto cruzado entre las bebidas y de descanso para los voluntarios. Seguidamente cada grupo fue expuesto a diferentes bebidas siguiendo los mismos procedimientos y tiempos (17) (Figura 1).

\section{Microdureza}

Los especímenes fueron llevados a un microdurómetro de Vickers HV 1000 (Liangong Group, Shandong, China). La MSV se midió antes y después de la exposición a las bebidas. El equipo fue calibrado en $50 \mathrm{~g}$ de presión durante 5 segundos (2). Se registraron tres medidas de microdureza por cada espécimen. Se calculó la diferencia de dureza superficial. Para el cálculo del porcentaje de pérdida de dureza se utilizó la siguiente fórmula $(\% \mathrm{PPD}=[($ Dureza final- dureza inicial) / dureza inicial] * 100) (12).

Los datos obtenidos fueron analizados en el programa SPSS versión 19 (IBM, Nueva York, Estados Unidos). Se realizó una análisis univariado (media y desviación estándar). Para el análisis bivariado se empleó el test ANOVA/Tukey $(\mathrm{a}=0,05)$.

\section{5 participantes}

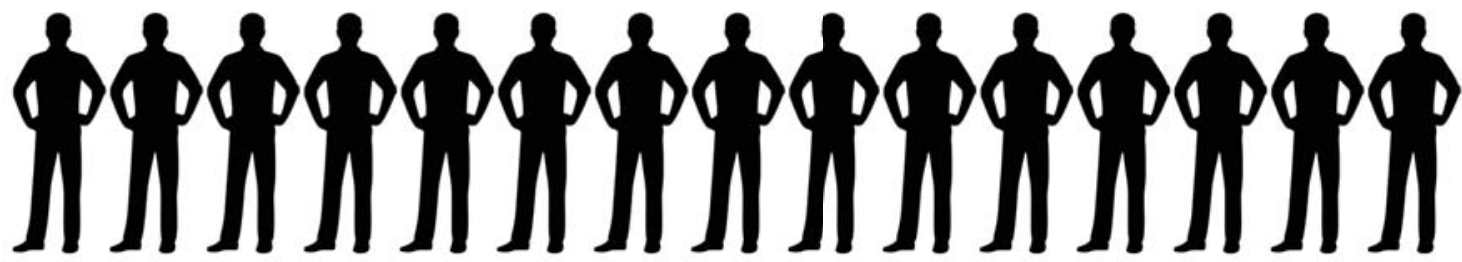

1 placa palatina / por participante

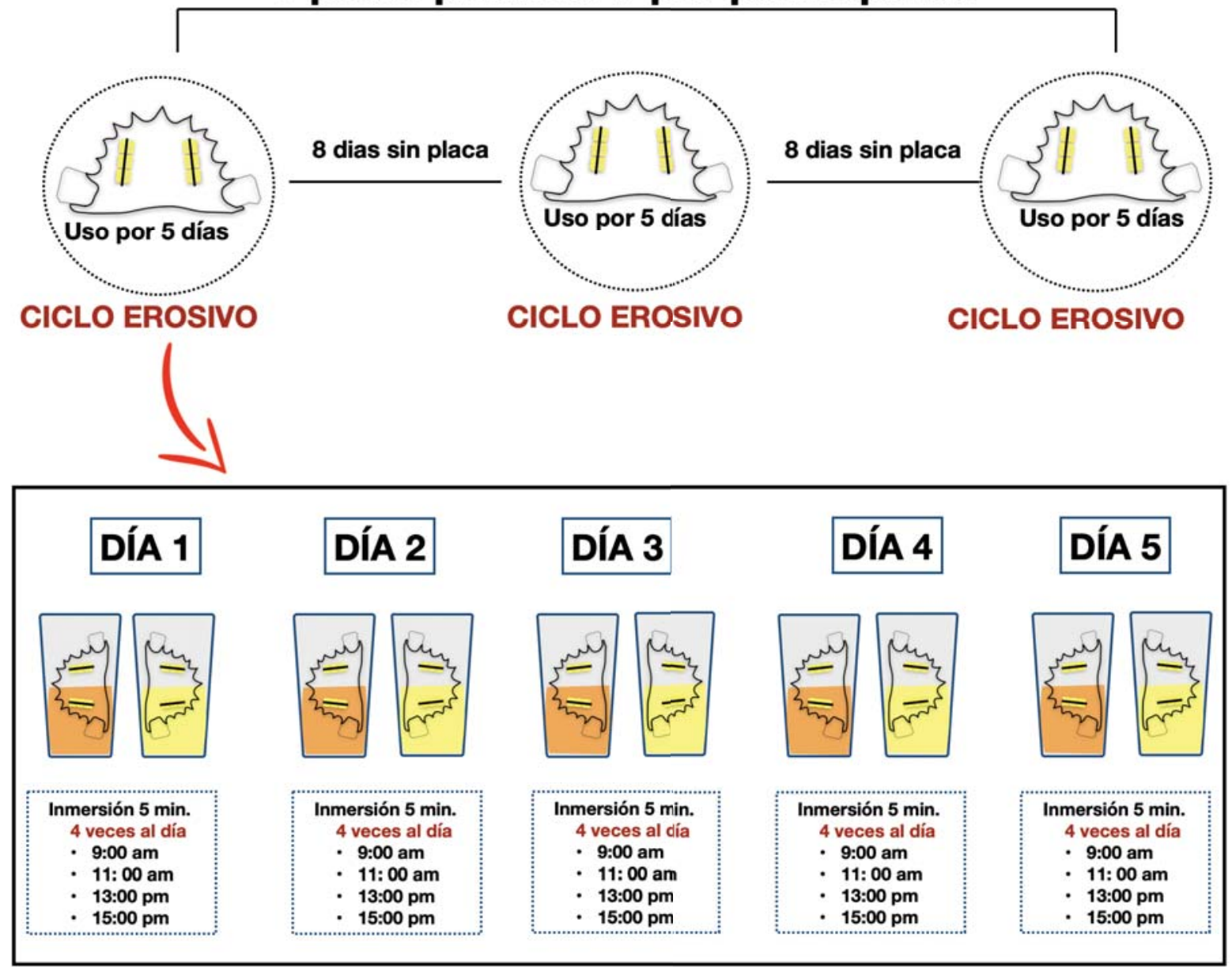

Figura 1. Esquema de metodología 


\section{RESULTADOS}

Los valores de microdureza superficial del esmalte inicial y final de acuerdo a los jugos utilizados se observan en la Tabla 1. Los cuatro grupos experimentales disminuyeron significativamente la microdureza superficial del esmalte $(p<0,05)$, sin diferencias significativas entre ellos (Gráfico 1).
Tabla 1. Media y desviacion estandar de los valores de microdureza $\left(\mathrm{kg} / \mathrm{mm}^{2}\right)$ de esmalte antes y después de la exposición a las bebidas

\begin{tabular}{lll}
\hline BEBIDAS & Inicial (DS) & Final (DS) \\
\hline Aguaymanto & $321,9(29,7)$ & $239,9(65,7)$ \\
Carambola & $321,9(29,0)$ & $242,8(58,0)$ \\
Camu camu & $323,0(28,6)$ & $230,9(54,7)$ \\
Coca cola & $322,0(27,5)$ & $212,0(80,5)$ \\
Agua destilada & $321,2(26,1)$ & $285,0(51,8)$ \\
\hline
\end{tabular}

Unidad de medición $\mathrm{kg} / \mathrm{mm}^{2}$

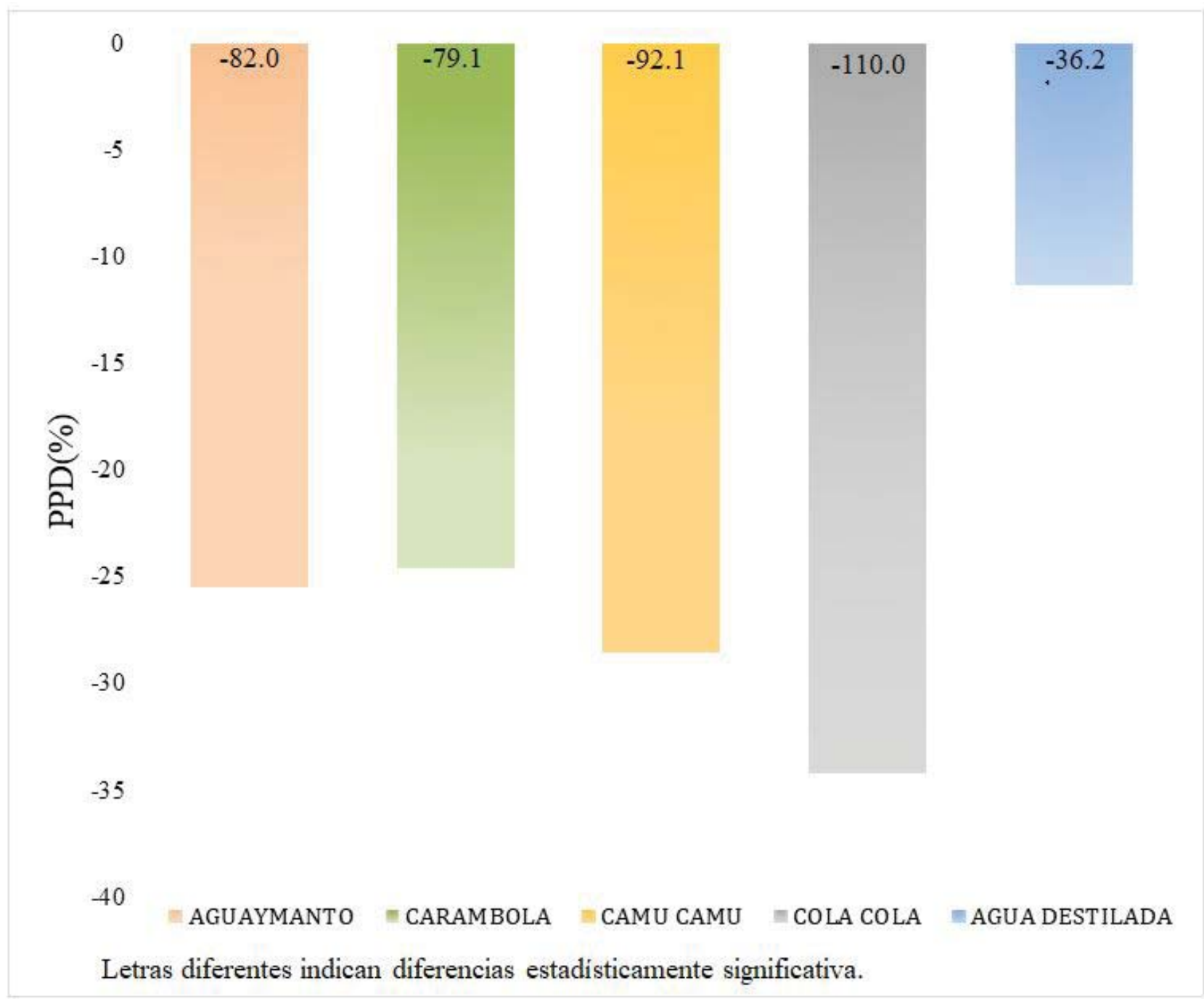

Gráfico 1. Porcentaje de pérdida de microdureza del esmalte dental expuesto a las distintas bebidas. 


\section{DISCUSIÓN}

El objetivo de este trabajo fue evaluar in situ el potencial erosivo de frutas amazónicas Physalis Peruviana (aguaymanto), Averrhoa Carambola (carambola), Myrciaria Dubia (camu camu) sobre el esmalte dental. Se encontró que todas las bebidas evaluadas produjeron una disminución de la microdureza superficial en el esmalte, similar a la Coca cola.

Se ha reportado que frutas tropicales como araçá boi, camu camu, cupuaçu, taperebá y umbu tienen el potencial erosivo en el esmalte evidenciando valores de pérdida de microdureza de 77,9 \% (araçá boi), 65,3\% (camu camu), 53,8\% (cupuaçu), 74,8\% (taperebá) y $77,3 \%$ (umbu) en un modelo in vitro (12).

El potencial erosivo de una bebida está relacionado al $\mathrm{pH}$ y la AT de la misma. El $\mathrm{pH}$ se refiere a la concentración de iones de hidrógeno $(\mathrm{H}+)$ en una solución y nos indica si una solución es ácida, neutra o básica. La AT corresponde a la cantidad de hidróxido de sodio $(\mathrm{NaOH})$ requerida para llevar a una solución a pH neutro $(5,11)$. Los jugos estudiados presentaban un $\mathrm{pH}$ ácido: aguaymanto (pH 3,7/AT 1,6-2,0), carambola (pH 1,98-2,16/AT 0,72-0,78) y camu camu ( $\mathrm{pH} 2,8-2,9 / \mathrm{AT} 1,08)$ presentando potencial erosivo $(12,13,24-26)$.

La exposición repetida a bebidas con un $\mathrm{pH}$ menor a 4,5 por cortos períodos de tiempo para el esmalte dental es un factor de riesgo para erosión, a diferencia de la lesión cariosa que implica la interacción con ácidos bacterianos de la placa durante periodos prolongados de tiempo generalmente a un $\mathrm{pH}$ superior a 5,5 (27).

Nirmala et al. compararon el $\mathrm{pH}$ y los oligoelementos de diferentes jugos de frutas y su relación con la erosión de esmalte, encontrando potencial erosivo de los jugos de piña ( $\mathrm{pH} 3,30)$, uva $(\mathrm{pH} 3)$, caña de azúcar ( $\mathrm{pH} 4,20)$, naranja ( $\mathrm{pH} 3,26)$, limón dulce ( $\mathrm{pH}$ $3,50)$, mango ( $\mathrm{pH} 4,20)$, granada ( $\mathrm{pH} 3,06)$, manzana $(\mathrm{pH} 5,25)$, zapote $(\mathrm{pH} 4,80)$ y sandía $(\mathrm{pH} 3,80)$ todos con un pH bajo; además los jugos de uva, piña y caña carecian de calcio en su composición por lo que no protegen la matriz del esmalte ante el ataque de bebidas ácidas (28).
La AT es otro factor físico-químico a considerar en el potencial erosivo. Enam et al. determinaron el potencial erosivo de gaseosas, bebidas energéticas, jugos de fruta $(\mathrm{pH}$ 2,4-3,9). La AT fue alta para las bebidas energéticas, moderado para gaseosas o jugos de frutas y bajo para agua. Cabe resaltar que la concentración de calcio fue mayor para los jugos de frutas (29). Benjakul y Chuenarrom encontraron que las bebidas de carabao y de guayaba presentaban un pH idéntico de 3,71, sin embargo la AT del carabao fue de 5,4 y de la guayaba 1,7. El carabao tuvo un mayor potencial erosivo que el jugo de guayaba con una pérdida de minerales del esmalte de $10,1 \%$ para la bebida de carabao y $6,9 \%$ para el jugo de guayaba (11). Así, cuanto mayor sea la cantidad de solución de $\mathrm{NaOH}$ añadida para alcanzar un valor de $\mathrm{pH}$ neutro, mayor será el efecto erosivo (30).

Los jugos evaluados en la presente investigación presentan ácido ascórbico. Se ha demostrado que diferentes ácidos presentan diferente potencial erosivo. Los ácidos maleico, tartárico y cítrico son los ácidos menos erosivos, mientras que los ácidos fosfórico, ascórbico y láctico son los más erosivos (30).

En relación al camu camu, Veras encontró que la pérdida de microdureza superficial del esmalte fue de $63,5 \%$ (12) y Ruilova de $77,58 \%$ (13) en modelos in vitro, sin embargo, en este estudio in situ se obtuvo una pérdida de $28,5 \%$. Se ha reportado que la Coca cola ocasionó una pérdida de microdureza superficial del esmalte de 77,27\% (pH 2.5/AT 0.1) que difiere de nuestro estudio con una pérdida de 34,2\% (31). Las diferencias se pueden explicar a los diferentes diseños metodológicos empleados para estudiar erosión dentaria, in vitro e in situ.

Los estudios in vitro se ejecutan en un periodo de tiempo corto, son más económicos, y proporcionan información al investigador antes de realizar estudios en el entorno clínico, sin embargo, sobreestiman la pérdida de tejido superficial por la falta de remineralización de la saliva artificial, carece de proteínas, pérdida de su capacidad protectora contra la degradación ácida, reducción de grado de amortiguación y descomposición de proteínas (17,32-34).

Los estudios in situ tienen muchas ventajas como un número reducido de voluntarios, cambios medibles en escala de tiempo más corto, posibilidad de controlar 
el desafío erosivo porque la desmineralización se produce fuera del medio bucal evitando daños perjudiciales a los tejidos de los voluntarios $(34,35)$ y el almacenamiento de los especímenes al medio bucal, donde la saliva puede diluir, neutralizar y amortiguar los ácidos. Además, la saliva transporta los iones calcio, fosfato y fluoruro al esmalte dental y desempeña un papel importante en la formación de la película adquirida (36), que actúa como una barrera semipermeable entre la superficie del diente y la cavidad oral, modulando los procesos de mineralización/desmineralización (15).

A pesar de las ventajas de los estudios in situ se han propuesto ciclos erosivos variados. Considerando que las lesiones erosivas iniciales se generan en tiempos cortos los especímenes han sido sumergidos durante 30 o 120 segundos, 4 veces al día durante 5 o 7 días $(15,20,21)$. También se han empleado exposiciones a bebidas erosivas de 5 minutos a 10 minutos, 4 veces al día durante 5-7 días (20,36,37). En nuestro estudio se expuso el esmalte por 5 minutos, 4 veces al día (20 minutos/ día) lo cual nos permite simular la ingesta regular de individuos de alto riesgo de erosión dental (36).

El intervalo entre cada desafío erosivo se estableció en dos horas para garantizar el efecto protector contra la erosión y la acción de remineralización $(19,20)$.

Por otro lado, se optó por el uso de placas palatinas y no linguales debido a la mayor evidencia de erosión en especímenes de placas palatinas y por la mayor comodidad de los participantes $(15,21)$. El uso de alambres de ortodoncia sobre los especímenes para inhibir el contacto con la lengua es importante ya que se ha demostrado que la pérdida de esmalte se incrementa por la combinación del consumo de bebidas ácidas y los efectos abrasivos de la lengua (22).

Los jugos de frutas estudiados se consideran saludables y se recomienda su consumo debido a su alto contenido de vitamina $\mathrm{C}$, vitamina $\mathrm{A}$, propiedades antioxidantes, antiinflamatorios y antimicrobiales, además son una alternativa para el tratamiento de diabetes, obesidad y enfermedades cardiovasculares $(38,39)$. Sin embargo, el consumo excesivo puede tener efectos perjudiciales en los tejidos dentales. Se ha detectado la aparición temprana de lesiones erosivas en jóvenes de 12 años de 6 comunidades con una correlación positiva entre la frecuencia de erosión dental y la proximidad de las plantaciones de cítricos, que presumiblemente se relacionó con el alcance del consumo diario de naranja (40). Por otro lado, existe una controversia respecto al consumo de jugos de frutas al $100 \%$ ya que los resultados combinan datos de jugos de frutas naturales y envasadas con aditivos de azúcar. Sin embargo, la evidencia existente sobre el consumo de fruta al $100 \%$ y erosión dental en estudios de asociación con factores de riesgo no es concluyente (6).

Es importante reconocer que para personas que incluyen en su alimentación frutos o bebidas a base de frutas con potencial erosivo deben tener controles periódicos para identificar lesiones erosivas iniciales. La intervención temprana puede ayudar a crear conciencia en pacientes y profesionales de la salud sobre los riesgos potenciales. También es importante combinar estrategias de prevención como el consumo de productos ricos en minerales de calcio y fosfato, estimulación salival, uso de productos a base de flúor, CPP-ACP, té verde y mejorar la higiene bucal $(5,13,27)$.

En conclusión, este estudio in situ demostró que los jugos a base de camu camu, aguaymanto y carambola son potencialmente erosivos.

\section{Correspondencia:}

Shareen Acuña

Correo electrónico: shareen.acuna@upch.pe

\section{REFERENCIAS BIBLIOGRÁFICAS}

1. Schlueter N, Amaechi BT, Bartlett D, et al. Terminology of Erosive Tooth Wear: Consensus Report of a Workshop Organized by the ORCA and the Cariology Research Group of the IADR. Caries Res. 2020;54(1):2-6.

2. de-Melo MA, Passos VF, Lima JP, Parente GC, Rodrigues LK, Santiago SL. Erosive potential of processed and fresh orange juice on human enamel. J Dent Child. 2015; 82(1):10-15.

3. Salas MM, Nascimento GG, Huysmans MC, Demarco FF. Estimated prevalence of erosive tooth wear in permanent teeth of children and adolescents: an epidemiological systematic review and metaregression analysis. J Dent. 2015;43(1):42-50.

4. Schlueter N, Luka B. Erosive tooth wear - a review on global prevalence and on its prevalence in risk groups. Br Dent J. 2018;224(5):364-370.

5. Buzalaf MAR, Magalhães AC, Rios D. Prevention of erosive tooth wear: targeting nutritional and patient- 
related risks factors. Br Dent J. 2018 ;224(5):371-78.

6. Ali H, Tahmassebi JF. The effects of smoothies on enamel erosion: an in situ study. Int J Paediatr Dent. 2014;24(3):184-91.

7. Liska D, Kelley M, Mah E. 100\% Fruit juice and dental health: A systematic review of the literature. Front Public Health. 2019;7:190.

8. Ruilova CE, León DC, Tay-Chu-Jon LY. Potencial erosivo de jugos naturales, jugos industrializados y gaseosas: Revisión de Literatura. Rev Estomatol Herediana . 2018; 28( 1 ): 56-63. DOI: 10.20453/reh. v28i1.3283

9. Lussi A, Megert B, Shellis RP, Wang X. Analysis of the erosive effect of different dietary substances and medications. Br J Nutr. 2012;107(2):252-62.

10. Lussi A, Jaeggi T, Zero D. The role of diet in the aetiology of dental erosion. Caries Res. 2004;38 Suppl 1:34-44.

11. Benjakul P, Chuenarrom C. Association of dental enamel loss with the $\mathrm{pH}$ and titratable acidity of beverages. J Dent Sci.2011; 6(3):129-33.

12. Veras AGC. Avaliação do potencial erosivo de sucos de frutas tropicais brasileiras.Tesis Doctoral. Piracicaba, SP: Universidade Estadual de Campinas, Faculdade de Odontologia de Piracicaba; 2011.

13. Ruilova CE. Evaluación del efecto neutralizante del té verde en el jugo de Camu Camu sobre la microdureza del esmalte dental.Tesis Especialidad] Lima: Universidad Peruana Cayetano Heredia, Facultad de Estomatología Roberto Beltrán Neira; 2018.

14. West NX, Davies M, Amaechi BT. In vitro and in situ erosion models for evaluating tooth substance loss. Caries Res. 2011;45:43-52.

15. Jordão MC, Ionta FQ, Bergantin BTP, et al. Influence of mandibular and palatal intraoral appliances on erosion in situ study outcome. J Appl Oral Sci. 2019;27:e53.

16. Almeida M. Estabelecimento de protocolos in vitro e in situ para estudos de erosão dentária.Tesis Doctoral. Bauru:, Faculdade de Odontologia de Bauru; 2016.

17. Hara AT, Ando M, González-Cabezas C, Cury JA, Serra MC, Zero DT. Protective effect of the dental pellicle against erosive challenges in situ. J Dent Res. 2006 ;85(7):612-6.

18. Hara AT, Barlow AP, Eckert GJ, Zero DT. Novel insitu longitudinal model for the study of dentifrices on dental erosion-abrasion. Eur J Oral Sci. 2014;122(2): 161-67.

19. Mendonça FL, Jordão $M C$, Ionta $F Q$, et al. In situ effect of enamel salivary exposure time and type of intraoral appliance before an erosive challenge. Clin Oral Investig. 2017; 21(8): 2465-71

20. Mendonça FL, Jordão MC, Val PP, et al. Eroded enamel rehardening using two intraoral appliances designs in different times of salivary exposure. J Clin Exp Dent. 2019; 11(12): e1127-32.
21. Santos NM, Jordão $M C$, Ionta $F Q$, et al. Impact of a simplified in situ protocol on enamel loss after erosive challenge. PLoS One.2018;13(5): e0196557.

22. Seong J, Claydon N, Macdonald E, Garner S, Newcombe RG, West N. A randomised clinical trial to determine the abrasive effect of the tongue on human enamel loss with and without a prior erosive challenge. J Dent. 2017;58: 48-53.

23. Ferreira A. Avaliação de sucos de frutas sobre materiais restauradores utilizados em lesoes vervicais ñao cariosas. Tesis Doctoral. Sao Paulo, Brasil: Universidad de Sao Paulo; 2013.

24. Arteaga H, León J. Estimación de modelos de regresión entre el color y las propiedades fisicoquímicas del aguaymanto (Physalis peruviana L.) Tecnología y Desarrollo. 2015;13(1): 35-40.

25. Mendoza J, Rodríguez A, Millán P. Caracterización físico química de la uchuva (Physalis peruviana) en la región de Silvia Cauca. Biotecnol sector agropecuario agroind [Internet] 2012; 10(2): 188-96. (Citado el 27 de mayo del 2020)Disponible en:

https://revistas.unicauca.edu.co/index.php/ biotecnologia/article/view/251

26. Tello O, García R, Vásquez O. Conservación de Averrhoa carambola L "carambola" por azúcar y calor. Revista Amazónica de Investigación Alimentaria. 2002;2 (1): 49-58. (Citado el 27 de mayo del 2020) Disponible en:

https://www.academia.edu/28012835/ CONSERVACI\%C3\%93 N_DE_Averrhoa_ c a r a m b o l a C A R A M B O L A P O R AZ\%C3\%9ACAR_Y CALOR

27. Faller RV, Noble WH. Protection from dental erosion: All Fluorides are Not Equal. Compend Contin Educ Dent. 2018;39(3):e13-e17.

28. Nirmala SV, Subba-Reddy VV. A comparative study of $\mathrm{pH}$ modulation and trace elements of various fruit juices on enamel erosion: an in vitro study. J Indian Soc Pedod Prev Dent. 2011;29(3):205-215.

29. Enam F, Mursalat M, Guha U, et al. Dental erosion potential of beverages and bottled drinking water in Bangladesh. International journal of food properties. 2017; 20(11):2499-2510.

30. Beyer M, Reichert J, Bossert J, Sigusch, BW, Watts DC, Jandt KD. Acids with an equivalent taste lead to different erosion of human dental enamel. Dent Mater.2011;27(10): 1017-1023.

31. de-Carvalho SH, Magalhães AC, de-Andrade-Moreira o MA, Buzalaf MA. Evaluation of the erosive potential of soft drinks. Eur J Dent. 2007;1(1):10-13.

32. Farooq I, Bugshan A. The role of salivary contents and modern technologies in the remineralization of dental enamel: a narrative review Version 2. F1000 Res. 2020;9:171.

33. Batista GR, Rocha-Gomes C, Sener B, Attin, T, Wiegand 
A. Artificial saliva formulations versus human saliva pretreatment in dental erosion experiments. Caries Res. 2016;50(1), 78-86.

34. West NX, He T, Hellin N, et al. Randomized in situ clinical trial evaluating erosion protection efficacy of a $0.454 \%$ stannous fluoride dentifrice. Int J Dent Hyg. 2019;17(3):261-67.

35. Creeth JE, Burnett GR, Souverain A, et al. In situ efficacy of an experimental toothpaste on enamel rehardening and prevention of demineralisation: a randomised, controlled trial. BMC Oral Health. 2020:20(1): 118

36. Rios D, Honório HM, Magalhães AC, et al. Effect of salivary stimulation on erosion of human and bovine enamel subjected or not to subsequent abrasion: an in situ/ex vivo study. Caries Res. 2006;40(3):218-23.

37. Ren YF, Liu X, Fadel N, Malmstrom H, Barnes V, $\mathrm{Xu}$ T. Preventive effects of dentifrice containing $5000 \mathrm{ppm}$ fluoride against dental erosion in situ. J Dent. 2011;39(10):672-78.
38. Arellano-Acuña E, Rojas-Zavaleta Irvin, PaucarMenacho L. Camu-camu (Myrciaria dubia): Fruta tropical de excelentes propiedades funcionales que ayudan a mejorar la calidad de vida. Scientia Agropecuaria. 2016 ;7(4):433-43. . (Citado el 27 de mayo del 2020)Disponible en: http://www.scielo. org.pe/scielo.php?script=sci_arttext\&pid=S2077$99172016000500008 \& \operatorname{lng}=\mathrm{es} \& \mathrm{nrm}=$ iso.

39. Muthu N, Lee SY, Phua KK, Bhore SJ. Nutritional, Medicinal and Toxicological Attributes of Star-Fruits (Averrhoa carambola L.): A Review. Bioinformation. 2016; 12(12): 420-24.

40. Künzel W, Cruz MS, Fischer T. Dental erosion in Cuban children associated with excessive consumption of oranges. Eur J Oral Sci. 2000;108(2):104-9.

Recibido: 15-07-20

Aceptado: 21-09-20 